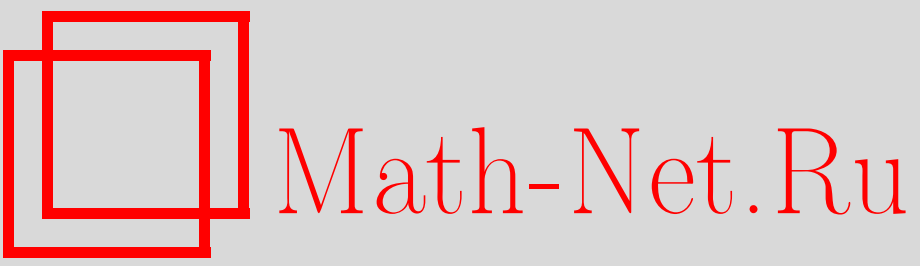

С. В. Бочкарев, Всюду расходящиеся ряды Фурье по системе Уолша и мультипликативным системам, УМH, 2004, том 59, выпуск 1, 103-124

DOI: https://doi.org/10.4213/rm703

Использование Общероссийского математического портала Math-Net.Ru подразумевает, что вы прочитали и согласны с пользовательским соглашением

http://www . mathnet.ru/rus/agreement

Параметры загрузки:

IP : 54.162 .85 .209

26 апреля 2023 г., 17:47:06 


\title{
ВСЮДУ РАСХОДЯЩИЕСЯ РЯДЫ ФУРЬЕ ПО СИСТЕМЕ УОЛША И МУЛЬТИПЛИКАТИВНЫМ СИСТЕМАМ
}

\author{
C. В. БОЧКАРЕВ
}

В статье изложена новая конструкция построения всюду расходящихся рядов Фурье-Уолша, которая позволила вдвое сократить разрыв в классах Лебега-Орлича между нижней оценкой Шиппа и Муна, установленной на основе конструкции Колмогорова, и верхней оценкой Сьёлина, полученной по методу Карлесона. Построены всюду расходящиеся после перестановки ряды Фурье по системе Уолша, а также по более общим системам характеров, с наилучшей нижней оценкой множителя Вейля. Установлены некоторые результаты, относящиеся к верхней оценке мажоранты частных сумм рядов по переставленньп мултипликативным системам. Полученные результаты показьвают определенные преимущества гармонического анализа на диадической группе для выявления и преодоления принципиальных трудностей в решении основных проблем анализа Фурье.

Библиографиия: 37 названий.

\section{СОДЕРЖАНИЕ}

$\S 1$. Всюду расходящиеся ряды Фурье по системе Уолша

$\S 2$. Всюду расходящиеся ряды по мультипликативным системам и смеж-

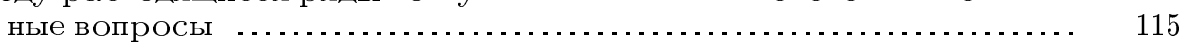

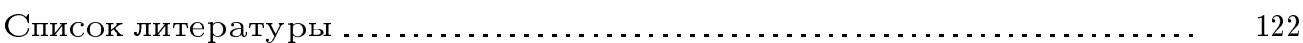

Исследование сходимости почти всюду и безусловной сходимости почти всюду рядов по классическим и общим ортонормированным системам является одной из центральных проблем современной теории рядов Фурье.

Фундаментальные результаты в этом направлении принадлежат А. Н. Колмогорову [1]-[4], Д. Е. Меньшову [5], [6], Л. Карлесону [7].

Теорема (А.Н. Колмогоров, 1926). Существует тригонометрический ряд Фурье-Лебега, расходящийся всюду.

Сходимость почти всюду рядов Фурье в классах Лебега-Орлича для тригонометрической системы и системы Уолша, а также для более обших систем характеров была установлена на основе метода Л. Карлесона в работах Р. Ханта [8], [9], П. Сьёлина [10], Дж. Госселина [11] и др.

Для системы Уолша наиболее сильной в настояшее время является следуюшая теорема [12].

Работа выполнена при финансовой поддержке Российского фонда фундаментальных исследований (грант № 02-01-00787), программы "Ведущие научные школы” (грант № 1549.2003.1) и гранта ОМН РАН "Современные проблемы теоретической математики”.

(C) С. В. БочКАрев 2004 
Теорема (П. Сьёлин, $\Phi$. Сориа). Если

$$
\int_{0}^{1}|f(x)| \log ^{+}|f(x)| \log ^{+} \log ^{+} \log ^{+}|f(x)| d x<\infty,
$$

то ряд Фурье-Уолша функции $f$ сходится почти всюду.

Расходящиеся ряды Фурье по системе Уолша и мультипликативным системам были построены И. Стейном [13], Ф. Шиппом [14], [15], К. Муном [16], П. Симоном [17] и др.

Теорема (Ф. Шипп, К. Мун). Для любого $\varepsilon>0$ существует такая функиия $f \in L\left(\log ^{+} \log ^{+} L\right)^{1-\varepsilon}$, ряд Фурье-Уолша которой расходится всюду.

Автору удалось распространить основную идею конструкции Колмогорова - полное устранение интерференции ядер Дирихле, соответствуюших различным $\delta$-функциям, на произвольные ограниченные ортонормированные системы [18]. Следует отметить, что главная трудность здесь состояла в отсутствии явного вида ядер Дирихле.

ОСНовНАЯ ЛЕммА (С.В. Бочкарев). Пусть $\left\{f_{n}(t)\right\}_{1}^{\infty}$ - ортонормированная система, удовлетворяющая условию

$$
\left\|f_{n}\right\|_{\infty} \leqslant M
$$

Тогда для любого $N=1,2, \ldots$ существуют множество $\Omega$ точек $\left(t, \theta_{1}, \ldots, \theta_{N}\right)$ единичного куба в $\mathbb{R}^{N+1}$,

$$
\operatorname{mes} \Omega \geqslant \gamma(M) \text {, }
$$

и последовательность натуральных чисел $\left\{m_{p}(t)\right\}, N p \leqslant m_{p}(t)<N(p+1)$, зависящая от $t$, такие, что для каждой точки $\left(t, \theta_{1}, \ldots, \theta_{N}\right) \in \Omega$ справедливо неравенство

$$
\varlimsup_{p \rightarrow \infty} \sum_{i=1}^{N} \sum_{n=N p}^{m_{p}(t)} f_{n}(t) f_{n}\left(\theta_{i}\right) \geqslant B N \log N
$$

где Ви $\gamma(M)$ - положительные постояннье.

На основании этого утверждения была установлена следующая теорема (см. [18]).

Теорема (С.В. Бочкарев). Для любой ортонормированной системы, ограниченной в совокупности, существует ряд Фурье-Лебега, расходящийся на множестве положительной мерь.

Как показал К. С. Казарян [19], множество положительной меры здесь нельзя заменить на множество полной меры даже для полных ортонормированных систем. Отметим, однако, что для систем характеров (тригонометрической системы, системы Уолша, мультипликативных систем и др.) из этой теоремы непосредственно следует сушествование рядов Фурье-Лебега, расходяшихся почти всюду.

Что касается количественных результатов, то прямое применение подхода Колмогорова для определения границы в классах Орлича между сходимостью и расходимостью почти всюду рядов Фурье дает только оценки с двойным логарифмом [14]-[16].

В этой статье содержится принципиально новая конструкция построения всюду расходяшихся рядов Фурье-Уолша, которая позволяет вдвое сократить разрьв между верхней оценкой Сьёлина и нижними оценками Шиппа и Муна. Кроме того, здесь 
построены всюду расходящиеся после перестановки ряды по системе Уолша и мультипликативным системам с наилучшей нижней оценкой множителя Вейля (см. [20]).

В истории проблемы безусловной сходимости почти всюду рядов по классическим и обшим ортогональным системам значительную роль сьграла работа Колмогорова и Меньшова [4], в которой была изложена новая конструкция построения почти всюду расходящихся ортогональных рядов и содержалась принадлежашая Колмогорову важная теорема о сушествовании тригонометрического ряда из $L_{2}$, расходящегося почти всюду после перестановки. Впоследствии эта теорема была обобщена на полные ортонормированные системы П. Л. Ульяновым [21] и А. М. Олевским [22].

Прогресс в определении скорости роста множителя Вейля для безусловной сходимости почти всюду был достигнут сначала для системы Уолша. Наилучшая в настоящее время и, вероятно, окончательная нижняя оценка множителя Вейля для безусловной сходимости почти всюду по системе Уолша $(\rho(n)$ порядка $\log n)$ была впервые установлена автором (см. [23]-[25]). Отметим, что несколько позже такую оценку с применением другой конструкции получил С. Наката [26].

В настоящей статье содержится усиление результатов автора по безусловной сходимости почти всюду, заключающееся в построении всюду расходящихся после перестановки рядов Фурье по системе Уолша и системам характеров. Кроме того, здесь содержатся некоторые результаты, относящиеся к оценке сверху мажоранты частных сумм для переставленных мультипликативных систем.

Обозначения: $\left\{r_{j}(x)\right\}_{j=0}^{\infty}$ - система функций Радемахера, определенных на всей вещественной оси и непрерьвных справа; $\left\{\psi_{n}(x)\right\}_{n=0}^{\infty}-$ система Уолша-Пэли, полная система характеров диадической группы; supp $f$ - носитель функции $f ; f^{+}$- положительная часть $f$; mes $E$ - мера Лебега множества $E$; $\log$ - натуральньй логарифм; $[x]$ - целая часть положительного числа $x$.

\section{$\S$ 1. Всюду расходящиеся ряды Фурье по системе Уолша}

Система Уолша - это классическая система ортонормированных функций, имеющая различные применения, в частности, к цифровой обработке информации. В контексте настоящей статьи необходимо подчеркнуть, что и система Уолша, и тригонометрическая система являются составной частью гармонического анализа на топологических группах, причем диадический гармонический анализ позволяет отчетливее выявить те принципиальные трудности, которые возникают на пути решения основных проблем анализа Фурье.

ТЕОрема 1. Пусть $\Phi(u)=u \varphi(u)$, әде $\varphi(u)$ - неубьвающая на $[0, \infty)$ функиия, $\varphi(0)=1$ и $\varphi(и)$ удовлетворяет условию

$$
\varphi(u)=o(\sqrt{\log u}) \quad n p u \quad u \rightarrow \infty
$$

Тогда существует такая функция $f \in \Phi(L)$, ряд Фурье-Уолша которой расходится всюду на $[0,1)$.

ДоКАЗАТЕЛЬСТВо. Используя (1), возьмем последовательность натуральных чисел $N_{\nu}$ и последовательность положительных чисел $\alpha_{\nu} \leqslant 1, \nu=1,2, \ldots$, для которых 
вьполняются соотношения

$$
\begin{gathered}
N_{\nu+1} \geqslant 2 N_{\nu} \\
\varphi(u) \leqslant \alpha_{\nu} \sqrt{\log u}, \quad \text { если } u \geqslant \frac{2^{N_{\nu}}}{\sqrt{N_{\nu}}}, \\
\sum_{\nu=1}^{\infty} \alpha_{\nu}<\infty .
\end{gathered}
$$

Для каждого $\nu=1,2, \ldots$ и $m=0,1, \ldots, 2^{N_{\nu}}-1$ определим полуинтервалы $\Delta_{m}^{\left(N_{\nu}\right)}$ и $\delta_{m}^{\left(N_{\nu}\right)}$ :

$$
\begin{aligned}
\Delta_{m}^{\left(N_{\nu}\right)} & =\left[\frac{m}{2^{N_{\nu}}}, \frac{m+1}{2^{N_{\nu}}}\right) \\
\delta_{m}^{\left(N_{\nu}\right)} & =\left[\frac{m}{2^{N_{\nu}}}+\frac{m}{2^{2 N_{\nu}}}, \frac{m}{2^{N_{\nu}}}+\frac{m}{2^{2 N_{\nu}}}+\frac{1}{2^{2 N_{\nu}}}\right)
\end{aligned}
$$

Теперь определим полиномы $P_{N_{\nu}}(x)$ по системе Уолша, на основе которых строится функция $f(x)$. Сначала положим

$$
F_{N_{\nu}}(x)=\frac{1}{\sqrt{N_{\nu}}} \sum_{j=0}^{N_{\nu}-1} r_{j}(x) .
$$

Полиномы $P_{N_{\nu}}(x)$ при $x \in[0,1)$ определим следуюшим соотношением $($ см. $(6),(7))$ :

$$
P_{N_{\nu}}(x)= \begin{cases}2^{N_{\nu}} F_{N_{\nu}}\left(\frac{m}{2^{N_{\nu}}}\right), & \text { если } x \in \delta_{m}^{\left(N_{\nu}\right)}, \\ 0, & \text { если } x \notin \bigcup_{m=0}^{\infty} \delta_{m}^{\left(N_{\nu}\right)} .\end{cases}
$$

Продолжим $P_{N_{\nu}}(x)$ периодически на $(-\infty, \infty)$.

Из (6)-(8) и неравенства Хинчина следует, что $\left\|P_{N_{\nu}}\right\|_{1} \asymp 1$ при всех $\nu=1,2, \ldots$.

Определим теперь функцию $f(x)$ в виде сходящегося в $L(0,1)$ ряда (см. $(2))$

$$
f(x)=\sum_{\nu=1}^{\infty} f_{\nu}(x),
$$

где

$$
f_{\nu}(x)=\frac{1}{\sqrt{N_{\nu}}} P_{N_{\nu}}\left(2^{2 N_{\nu}} x\right) .
$$

Для каждой точки $x \in[0,1)$ построим две последовательности натуральных чисел $n(\nu, x)$ и $q(\nu, x)$ такие, что

$$
2^{2 N_{\nu}} \leqslant q(\nu, x)<n(\nu, x)<2^{4 N_{\nu}} .
$$

Положим (см. (11))

$$
n(\nu, x)=\sum_{j=2 N_{\nu}}^{4 N_{\nu}-1} \varepsilon_{j}(x) 2^{j},
$$

где функщии $\varepsilon_{j}(x)$ принимают значения 0 или 1. 
Пусть теперь

$$
\begin{aligned}
& E_{1}=\left\{\sum_{j=2 N_{\nu}}^{3 N_{\nu}-1} r_{j}^{+}(x) \geqslant \frac{N_{\nu}}{2}\right\}, \\
& E_{2}=[0,1) \backslash E_{1} .
\end{aligned}
$$

Определим $\varepsilon_{j}(x)$ сначала при $2 N_{\nu} \leqslant j<3 N_{\nu}$, полагая

$$
\varepsilon_{j}(x)= \begin{cases}\frac{1}{2}\left(1+\operatorname{sign} r_{j}(x)\right), & \text { если } x \in E_{1}, \\ \frac{1}{2}\left(1-\operatorname{sign} r_{j}(x)\right), & \text { если } x \in E_{2} .\end{cases}
$$

Затем при $3 N_{\nu} \leqslant j<4 N_{\nu}$ положим

$$
\varepsilon_{j}(x)=\varepsilon_{j-N_{\nu}}(x) .
$$

Числа $q(\nu, x)$ определим следующим образом:

$$
q(\nu, x)=\sum_{j=3 N_{\nu}}^{4 N_{\nu}-1} \varepsilon_{j}(x) 2^{j} .
$$

Используя модифицированное ядро Дирихле $D_{m}^{*}(x)($ см. [10], [27]) и операцию сложения на диадической группе, имеем

$$
S_{m}(f, x)=\sum_{i=0}^{m-1}\left(f, \psi_{i}\right) \psi_{i}(x)=\int_{0}^{1} f(t) \psi_{m}(x \oplus t) D_{m}^{*}(x \oplus t) d t .
$$

Так как при $q(\nu, x) \leqslant i<n(\nu, x)$ функции $\psi_{i}(x)$ ортогональны $f_{m}(x)$, если $m \neq \nu$ (см. (2), (10), (11)), то (см. (18))

$$
\begin{aligned}
& S_{n(\nu, x)}(f, x)-S_{q(\nu, x)}(f, x) \\
& \quad=\psi_{n(\nu, x)}(x) \sum_{j=2 N_{\nu}}^{3 N_{\nu}-1} \varepsilon_{j}(x) \int_{0}^{1} f_{\nu}(t) \psi_{n(\nu, x)}(t) D_{2^{j}}^{*}(x \oplus t) d t .
\end{aligned}
$$

Здесь учитьвалось, что при любом $t \in[0,1)$ для $j \geqslant 3 N_{\nu}$ ввиду соотношений $(12),(17)$ имеем

$$
\psi_{n(\nu, x)}(t) D_{2^{j}}^{*}(t)=\psi_{q(\nu, x)}(t) D_{2^{j}}^{*}(t) .
$$

Далее, при всех $t, x \in[0,1)$ справедливо равенство

$$
f_{\nu}(t) \psi_{n(\nu, x)}(t)=f_{\nu}(t)
$$

В самом деле (см. (12), (17)),

$$
\psi_{n(\nu, x)}(t)=\psi_{n(\nu, x)-q(\nu, x)}(t) \psi_{q(\nu, x)}(t)
$$

Но при $t \in \operatorname{supp} f_{\nu}$ в силу определения чисел $n(\nu, x)$ и $q(\nu, x)$ и функций $f_{\nu}(t)($ см. $(6)$, $(8),(10))$ вьполняется равенство

$$
\psi_{n(\nu, x)-q(\nu, x)}(t)=\psi_{q(\nu, x)}(t) .
$$

Отсюда следует (20). 
Ввиду соотношения (20), снова используя определение функции $f_{\nu}(t)$, получаем, что при любом $j<3 N_{\nu}$ справедливо равенство (см. (5), (6), (8), (10))

$$
\int_{0}^{1} f_{\nu}(t) \psi_{n(\nu, x)}(t) D_{2^{j}}^{*}(x \oplus t) d t=\frac{1}{N_{\nu}} \int_{0}^{1} G_{\nu}(t) D_{2^{j}}^{*}(x \oplus t) d t
$$

где

$$
G_{\nu}(t)=\sum_{j=2 N_{\nu}}^{3 N_{\nu}-1} r_{j}(t)
$$

Соединяя соотношения (19), (21) и (22), приходим к вьводу, что

$$
S_{n(\nu, x)}(f, x)-S_{q(\nu, x)}(f, x)=\frac{1}{N_{\nu}} \psi_{n(\nu, x)}(x) \sum_{j=2 N_{\nu}}^{3 N_{\nu}-1} \varepsilon_{j}(x) r_{j}(x) .
$$

В силу выбора функций $\varepsilon_{j}(x)$ (см. (15)) из (23) получаем окончательно, что при всех $\nu=1,2, \ldots$ и $x \in[0,1)$ имеет место оценка

$$
\left|S_{n(\nu, x)}(f, x)-S_{q(\nu, x)}(f, x)\right| \geqslant \frac{1}{2}
$$

Итак, установлено (см. (2), (11), (24)), что ряд Фурье-Уолша функции $f(x)$ всюду расходится.

Докажем, что $f \in \Phi(L)$. Обозначим

$$
E_{\nu}=\operatorname{supp} f_{\nu}
$$

и положим

$$
G_{\nu}=E_{\nu} \backslash \bigcup_{m=\nu+1}^{\infty} E_{m}
$$

Из (6)-(10) следует, что $\lim _{\nu \rightarrow \infty} \operatorname{mes}\left(\bigcup_{m=\nu}^{\infty} E_{m}\right)=0$, и поэтому с точностью до множества меры нуль

$$
\operatorname{supp} f=\bigcup_{\nu=1}^{\infty} G_{\nu}
$$

Таким образом, учитывая, что $G_{\nu} \cap G_{m}=\varnothing$ при $m \neq \nu$, имеем (см. (9), (25))

$$
\int_{0}^{1} \Phi(|f(x)|) d x=\sum_{\nu=1}^{\infty} \int_{G_{\nu}} \Phi(|f(x)|) d x=\sum_{\nu=1}^{\infty} \int_{G_{\nu}} \Phi\left(\left|\sum_{m=1}^{\nu} f_{m}(x)\right|\right) d x
$$

Далее, $\operatorname{mes} G_{\nu} \leqslant 2^{-N_{\nu}}$ и, следовательно (см. (25), (26)),

$$
\int_{G_{\nu}} \Phi(|f(x)|) d x \leqslant \alpha_{\nu}+\int_{\alpha_{\nu} 2^{N_{\nu}}}^{\infty} \operatorname{mes}\left\{\Phi\left(\left|\sum_{m=1}^{\nu} f_{m}(x)\right|\right)>y\right\} d y .
$$


Так как $\Phi(u)$ строго возрастает, то существует неубьвающая обратная функция $\Psi(y)$. Поэтому, учитывая, что $\zeta(2)=\pi^{2} / 6$, получим

$$
\begin{gathered}
\int_{\alpha_{\nu} 2^{N_{\nu}}}^{\infty} \operatorname{mes}\left\{\Phi\left(\left|\sum_{m=1}^{\nu} f_{m}(x)\right|\right)>y\right\} d y \leqslant \int_{\alpha_{\nu} 2^{N_{\nu}}}^{\infty} \operatorname{mes}\left\{\left|\sum_{m=1}^{\nu} f_{m}(x)\right| \geqslant \Psi(y)\right\} d y \\
\leqslant \sum_{m=1}^{\nu} \int_{\alpha_{\nu} 2^{N_{\nu}}}^{\infty} \operatorname{mes}\left\{\left|f_{m}(x)\right| \geqslant \frac{1}{(\nu-m+2)^{2}} \Psi(y)\right\} d y
\end{gathered}
$$

Теперь для каждой функции $f_{m}$ на ее носителе $E_{m}$ применим экспоненциальную оценку для распределения суммы независимых случайных величин (см. [28; с. 93]). В силу этой оценки имеем (см. (6)-(10))

$$
\operatorname{mes}\left\{x \in E_{m} ;\left|f_{m}(x)\right| \geqslant \frac{\Psi(y)}{(\nu-m+2)^{2}}\right\} \ll \frac{1}{2^{N_{m}}} \exp \left(-\frac{1}{2} \cdot \frac{\Psi^{2}(y) N_{m}}{(\nu-m+2)^{4} 2^{2 N_{m}}}\right) .
$$

Для функции $\Psi(y)$ из (3) вьводим следующую нижнюю оценку:

$$
\Psi(y) \geqslant \frac{y}{\alpha_{\nu} \sqrt{\log y}} \text { при } y \geqslant \alpha_{\nu} 2^{N_{\nu}} .
$$

Соединим оценки (28)-(30) и преобразуем внутренний интеграл. Имеем

$$
\begin{aligned}
& \int_{\alpha_{\nu} 2^{N_{\nu}}}^{\infty} \operatorname{mes}\left\{\Phi\left(\left|\sum_{m=1}^{\nu} f_{m}(x)\right|\right)>y\right\} d y \\
& \ll \sum_{m=1}^{\nu} \frac{1}{2^{N_{\nu}}} \int_{\alpha_{\nu} 2^{N_{\nu}}}^{\infty} \exp \left(-\frac{y^{2} N_{m}}{2 \alpha_{\nu}^{2}(\nu-m+2)^{4} 2^{2 N_{m}} \log y}\right) d y \\
& \leqslant \alpha_{\nu} \sum_{m=1}^{\nu} \int_{2^{N_{\nu}-N_{m}}}^{\infty} \exp \left(-\frac{y^{2}}{2(\nu-m+2)^{4}(1+\log y)}\right) d y
\end{aligned}
$$

Отсюда, ввиду того что $(\nu-m+2)^{4}(1+\log y) \ll y$ при $y \geqslant 2^{N_{\nu}-N_{m}}$, находим окончательную оценку (см. (2), (27), (31))

$$
\int_{G_{\nu}} \Phi(|f(x)|) d x \ll \alpha_{\nu}
$$

Теперь из соотношений (4), (26) и (32) следует, что

$$
\int_{0}^{1} \Phi(|f(x)|) d x<\infty
$$

Теорема 1 доказана.

Предложенная конструкция построения всюду расходящихся рядов Фурье-Уолша позволяет установить следуюшие результаты. 
ТЕОРема 2. Если возрастающая последовательность положительных чисел $\left\{\lambda_{n}\right\}$ удовлетворяет условию

$$
\lambda_{n}=o(\sqrt{\log n}) \quad \text { npu } n \rightarrow \infty,
$$

то существует $f \in L(0,1)$ такая, что при всех $x \in[0,1)$

$$
\varlimsup_{n \rightarrow \infty} \frac{\left|S_{n}(f, x)\right|}{\lambda_{n}}=\infty
$$

Для тригонометрической системы несколько менее точные результаты, чем теоремы 1 и 2, установлены С. В. Конягиньм [29].

ТЕОРема 3. Существует такая $f \in L(0,1)$, что ее модуль непрерывности в $L_{1}$ удовлетворяет условию

$$
w_{1}(\delta, f)=o\left(\log \frac{1}{\delta}\right)^{-\frac{1}{2}},
$$

но ряд Фурье-Уолша функиии $f$ всюду расходится.

Теорема 3 усиливает результат автора [30], где рассматривалась расходимость почти всюду.

Обратимся теперь к проблеме безусловной сходимости.

ТЕОРема 4. Пусть неубывающая последовательность положительных чисел $\rho(k)$ удовлетворяет условию

$$
\sum_{k=1}^{\infty} \frac{1}{k \rho(k)}=\infty
$$

Тогда существуют последовательность коэффичиентов $\left\{a_{k}\right\}$ и перестановка натурального ряда $\sigma(k)$ такие, что

$$
\sum_{k=1}^{\infty} a_{k}^{2} \rho(k)<\infty
$$

но переставленный ряд Фурье-Уолиа $\sum_{k=1}^{\infty} a_{\sigma(k)} \psi_{\sigma(k)}(x)$ расходится при всех $x \in[0,1)$.

ДокАЗАТЕЛЬСТво. Для каждого $n=0,1,2, \ldots$, и $m=0,1, \ldots, 2^{n}-1$ определим специальные подгруппы $G_{n m}$ и смежные классы по этим подгруппам $H_{n m}$.

Положим

$$
H_{n m}=\psi_{q_{n m}} G_{n m}
$$

где

$$
\begin{gathered}
\psi_{q_{n 0}}=r_{2 n}, \quad \text { если } m=0 \\
\psi_{q_{n m}}=r_{2 n} r_{2 p_{1}+1} r_{2 p_{2}+1} \cdots r_{2 p_{s(m)}+1}
\end{gathered}
$$

если

$$
m=2^{p_{1}}+2^{p_{2}}+\cdots+2^{p_{s(m)}}, \quad 0 \leqslant p_{1}<p_{2}<\cdots<p_{s(m)}<n .
$$


Подгрупшу $G_{n m}$ зададим при $n \geqslant 1\left(G_{00}=\psi_{0}\right)$ образующими $\psi_{l_{n m}^{(k)}}, k=1, \ldots, n$, где $l_{n 0}^{(k)}=2(n-k)$ и

$$
l_{n m}^{(k)}=\left[2^{2(n-k)}+2^{2\left(p_{1}-k\right)+1}+2^{2\left(p_{2}-k\right)+1}+\cdots+2^{2\left(p_{s(m)}-k\right)+1}\right],
$$

если $m$ имеет разложение (38). Условимся считать, что

$$
r_{k}(x)=1 \text { при } k<0, \quad x \in[0,1) .
$$

Тогда образуюшие $\psi_{l_{n m}^{(k)}}$ можно записать следуюшим образом (см. (37)-(40)):

$$
\psi_{l_{n m}^{(k)}}=r_{2(n-k)} r_{2\left(p_{1}-k\right)+1} \cdots r_{2\left(p_{s(m)}-k\right)+1}
$$

Докажем, что все классы $H_{n m}$ не пересекаются. Прежде всего заметим, что классы $H_{n m}$ не могут пересекаться при разных $n$, так как если $p<n$, то функции класса $H_{n m}$ содержат в качестве сомножителя функцию $r_{2 n}(x)$, а функщии класса $H_{p q}$ эту функцию Радемахера не содержат (см. (35)-(39)).

Далее, при фиксированном $n$ и фиксированном $1 \leqslant p \leqslant n$ классы $H_{n m_{1}}$, где $0 \leqslant$ $m_{1}<2^{p-1}$, и $H_{n m_{2}}$, где $2^{p-1} \leqslant m_{2}<2^{p}$, не пересекаются, поскольку функция $r_{2 p-1}$ входит в качестве сомножителя в любую из функций класса $H_{n m_{2}}$, но не входит в функции класса $H_{n m_{1}}$.

Предположим теперь, что

$$
\begin{gathered}
\psi_{\alpha_{1}}=\psi_{\alpha_{2}} \\
\psi_{\alpha_{1}} \in H_{n m_{1}}, \quad \psi_{\alpha_{2}} \in H_{n m_{2}}, \\
m_{1}=2^{p-1}+\nu_{1}, \quad m_{2}=2^{p-1}+\nu_{2}, \quad 0 \leqslant \nu_{1}, \nu_{2}<2^{p-1} .
\end{gathered}
$$

Раскладьвая элементы подгруп $G_{n m_{1}}$ и $G_{n m_{2}}$ по образуюшим, имеем

$$
\begin{aligned}
& \psi_{\alpha_{1}}=\psi_{q_{n m_{1}}} \psi_{l_{n m_{1}}^{\left(k_{1}\right)} \cdots \psi_{l_{n m_{1}}^{\left(k_{i}\right)}}}, \\
& \psi_{\alpha_{2}}=\psi_{q_{n m_{2}}} \psi_{l_{n m_{2}}^{\left(s_{1}\right)} \cdots \psi_{l_{n m_{2}}^{\left(s_{j}\right)}}},
\end{aligned}
$$

где $1 \leqslant k_{1}<\cdots<k_{i} \leqslant n, 1 \leqslant s_{1}<\cdots<s_{j} \leqslant n$.

Каждая образуюшая подгруппы $G_{n m}$ содержит только одну функцию Радемахера с четньм номером (см. (41)), а именно функцию $r_{2(n-k)}$. Поэтому (см. $\left.(36),(37)\right)$ равенство $\alpha_{1}=\alpha_{2}$ возможно лишш в том случае, если $i=j, k_{1}=s_{1}, k_{2}=s_{2}, \ldots, k_{i}=s_{i}$. Но тогда (см. (35)-(41))

$$
\begin{aligned}
& \psi_{\alpha_{1}}=r_{2 p-1} r_{2\left(p-k_{1}\right)+1} \cdots r_{2\left(p-k_{i}\right)+1} \psi_{\beta_{1}}, \\
& \psi_{\alpha_{2}}=r_{2 p-1} r_{2\left(p-k_{1}\right)+1} \cdots r_{2\left(p-k_{i}\right)+1} \psi_{\beta_{2}},
\end{aligned}
$$

где $\psi_{\beta_{1}} \in H_{n \nu_{1}}, \psi_{\beta_{2}} \in H_{n \nu_{2}}$, т.е. из равенства $\psi_{\alpha_{1}}=\psi_{\alpha_{2}}$ следует, что $\psi_{\beta_{1}}=\psi_{\beta_{2}}$.

Таким образом, вопрос о сравнении классов $H_{n m_{1}}$ и $H_{n m_{2}}$ при $0 \leqslant m_{1}, m_{2}<2^{n}$ можно редуцировать к вопросу о сравнении классов $H_{n \nu_{1}}$ и $H_{n \nu_{2}}$, где $0 \leqslant \nu_{1}, \nu_{2}<$ $2^{n-1}$. Продолжая этот процесс, придем к сравнению классов $H_{n 0}$ и $H_{n 1}$. Но классы $H_{n 0}$ и $H_{n 1}$ не пересекаются, так как функции класса $H_{n 0}$ состоят из функций Радемахера с четными номерами, а функции класса $H_{n 1}$ содержат $r_{1}$. 
Итак, доказано, что

$$
H_{n m} \cap H_{n_{1} m_{1}}=\varnothing, \text { если }(n, m) \neq\left(n_{1}, m_{1}\right) .
$$

Определим множества $E_{m}^{(n)}$. Множество $E_{0}^{(0)}=[0,1)$. Пусть множества $E_{q}^{(p)}$ определены при $p \leqslant n, q=0,1, \ldots, 2^{p}-1$. Тогда (см. (37))

$$
\begin{aligned}
& E_{2 m}^{(n+1)}=\left\{x \in E_{m}^{(n)} ; \psi_{q_{n m}}(x)=1\right\}, \\
& E_{2 m+1}^{(n+1)}=\left\{x \in E_{m}^{(n)} ; \psi_{q_{n m}}(x)=-1\right\} .
\end{aligned}
$$

Из определений (43), (44) следует, что

$$
E_{m}^{(n)} \subset E_{m_{1}}^{(n-1)} \subset \cdots \subset E_{m_{k}}^{(n-k)} \subset \cdots \subset E_{0}^{(0)},
$$

где

$$
m_{k}=\left[\frac{m}{2^{k}}\right]
$$

Для каждого $N=0,1,2, \ldots$ определим функцию пары чисел $\lambda_{N}(n, m), n=$ $0,1, \ldots, N, m=0,1, \ldots, 2^{N}-1$. Положим

$$
\lambda_{N}(n, m)=\left(2^{n+1}-2 m-1\right) 2^{N-n} .
$$

Функция $\lambda_{N}(n, m)$ осуществляет взаимно однозначное отображение множества пар $(n, m)$ на отрезок натурального ряда от 1 до $2^{N+1}-1$.

Установим, что справедливо следуюшее утверждение. Пусть

$$
\lambda_{N}\left(n_{1}, m_{1}\right)>\lambda_{N}(n, m)
$$

и вьполняется соотношение

$$
E_{2 m+1}^{(n+1)} \cap E_{m_{1}}^{\left(n_{1}\right)} \neq \varnothing
$$

Тогда

$$
E_{2 m+1}^{(n+1)} \subset E_{2 m_{1}+1}^{\left(n_{1}+1\right)}
$$

Сначала докажем, что из (48), (49) следует, что $n_{1}<n$. В самом деле, если $n_{1}=n$, то, используя (43), (44) и учитьвая (49), получаем, что $m_{1}=m$, а это противоречит (48). Если же $n<n_{1}$, то согласно (45), (46) из (49) следует, что

$$
E_{m_{1}}^{\left(n_{1}\right)} \subseteq E_{2 m+1}^{(n+1)}
$$

и вьполняется соотношение

$$
2 m+1=\left[\frac{m_{1}}{2^{n_{1}-n-1}}\right] .
$$

Но, используя определение функции $\lambda_{N}(n, m)$ и условие (48), имеем (см. (47))

$$
2 m+1>\left(2 m_{1}+1\right) 2^{n-n_{1}}>\frac{m_{1}}{2^{n_{1}-n-1}}
$$

и, сопоставляя (51) и (52), получаем противоречие. 
Итак, $n_{1}<n$ и, следовательно (см. (43), (44), (49)),

$$
E_{2 m+1}^{(n+1)} \subset E_{q}^{\left(n_{1}+1\right)}
$$

где $q$ принимает одно из двух значений: $2 m_{1}, 2 m_{1}+1$.

Применяя (45), (46), получаем

$$
q=\left[\frac{2 m+1}{2^{n-n_{1}}}\right]
$$

Вместе с тем из (47), (48) следует неравенство

$$
\left[\frac{2 m+1}{2^{n-n_{1}}}\right] \geqslant 2 m_{1}+1
$$

Таким образом (см. (54)), $q=2 m_{1}+1$ и (см. (53)) соотношение (50) доказано.

Перейдем к определению коэффициентов $\left\{a_{k}\right\}$. Обозначим

$$
A_{n m}=\left\{k ; \psi_{k} \in H_{n m}\right\} .
$$

В силу соотношений (37), (41) и (43)-(46) любая функция из класса $H_{n m}$ принимает постоянное значение на множестве $E_{2 m}^{(n+1)}$. Положим (см. (55))

$$
\varepsilon_{k}=\operatorname{sign} \psi_{k}(x), \quad x \in E_{2 m}^{(n+1)} .
$$

Возьмем последовательность положительных чисел $\mu(n)$, для которой (см. (33))

$$
\begin{aligned}
& \sum_{n=1}^{\infty} \frac{\mu(n)}{\rho\left(2^{2 n+1}\right)}=\infty \\
& \sum_{n=1}^{\infty} \frac{\mu^{2}(n)}{\rho\left(2^{2 n+1}\right)}<\infty .
\end{aligned}
$$

Учитывая (см. (42), (55)), что $A_{n m} \cap A_{n_{1} m_{1}}=\varnothing$ при $(n, m) \neq\left(n_{1}, m_{1}\right)$, определим коэффициенты $\left\{a_{k}\right\}$ следуюшим образом (см. $\left.(56)\right)$ :

$$
a_{k}= \begin{cases}\frac{\mu(n)}{2^{n} \rho\left(2^{2 n+1}\right)} \varepsilon_{k} & \text { при } k \in A_{n m}, \\ 0 & \text { при остальных } k .\end{cases}
$$

Используя соотношения (55), (56), (58), (59), имеем (см. (37), (41))

$$
\sum_{k=1}^{\infty} a_{k}^{2} \rho(k)=\sum_{n=0}^{\infty} \frac{\mu^{2}(n)}{2^{2 n} \rho^{2}\left(2^{2 n+1}\right)} \sum_{m=0}^{2^{n}-1} \sum_{k \in A_{n m}} \rho(k) \leqslant \sum_{n=0}^{\infty} \frac{\mu^{2}(n)}{\rho\left(2^{2 n+1}\right)}<\infty .
$$

Таким образом, коэффициенты $\left\{a_{k}\right\}$ удовлетворяют условию (34). 
Пусть теперь $\left\{N_{\nu}\right\}$ - такая последовательность натуральных чисел, для которой (см. (57))

$$
\sum_{n=N_{\nu}+1}^{N_{\nu+1}} \frac{\mu(n)}{\rho\left(2^{2 n+1}\right)} \geqslant \nu .
$$

Обозначим (см. (59))

$$
S_{n m}(x)=\sum_{k \in A_{n m}} a_{k} \psi_{k}(x)
$$

В силу выбора коэффициентов $\left\{a_{k}\right\}$ и свойств классов $H_{n m}$ и множеств $E_{m}^{(n)}$ (см. (43)-(46), (55), (56), (59), (61)) имеем

$$
S_{n m}(x)= \begin{cases}\frac{\mu(n)}{\rho\left(2^{2 n+1}\right)} & \text { при } x \in E_{2 m}^{(n+1)}, \\ -\frac{\mu(n)}{\rho\left(2^{2 n+1}\right)} & \text { при } x \in E_{2 m+1}^{(n+1)}, \\ 0 & \text { при остальных } x \in[0,1) .\end{cases}
$$

Определим перестановку $\sigma(k)$. Суммы

$$
\sum^{(\nu)}=\sum_{n=N_{\nu}+1}^{N_{\nu+1}} \sum_{m=0}^{2^{n}-1} S_{n m}(x)
$$

расположим в порядке возрастания номера $\nu$.

Внутри сумм $\sum^{(\nu)}$ порядок слагаемых $S_{n m}(x)$ зададим, используя $\lambda_{N_{\nu+1}}(n, m)$. Для каждого фиксированного $\nu=1,2, \ldots$ возьмем функцию $\lambda_{N_{\nu+1}}(n, m)$, где $n=$ $0,1, \ldots, N_{\nu+1}, m=0,1, \ldots, 2^{n}-1$, и расположим суммы $S_{n m}(x)$, где $N_{\nu}<n \leqslant$ $N_{\nu+1}$, в порядке возрастания чисел $\lambda_{N_{\nu+1}}(n, m)$. Внутри суммы $S_{n m}(x)$ слагаемые расположим в порядке возрастания номеров функций Уолша.

При фиксированном $\nu$ для каждой точки $x \in[0,1)$ определим число $l(x)$. Положим

$$
l(x)=\min \lambda_{N_{\nu+1}}(n, m),
$$

где минимум берется по всем парам $(n, m), N_{\nu}<n \leqslant N_{\nu+1}$, таким, что $x \in E_{2 m+1}^{(n+1)}$.

Из доказанного ранее утверждения (см. (48)-(50)) следует, что $l(x)$ является тем номером, на котором расположенная в порядке возрастания чисел $\lambda_{N_{\nu+1}}(n, m)$ последовательность функщий $S_{n m}(x)$, отличных от нуля в точке $x$, меняет знак с плюса на минус (см. (61)-(63)).

Поэтому имеем

$$
\left|\sum_{\substack{N_{\nu}<n \leqslant N_{\nu+1} \\ \lambda_{N_{\nu+1}}(n, m)<l(x)}} S_{n m}(x)\right|+\left|\sum_{\substack{N_{\nu}<n \leqslant N_{\nu+1} \\ \lambda_{N_{\nu+1}}(n, m) \geqslant l(x)}} S_{n m}(x)\right|=\sum_{n=N_{\nu}+1}^{N_{\nu+1}} \sum_{m=0}^{2^{n}-1}\left|S_{n m}(x)\right| .
$$


Таким образом, получаем, что для любого $\nu=1,2, \ldots$ в каждой точке $x \in[0,1)$ вьполняется соотношение (см. (60), (62), (64))

$$
\begin{aligned}
& \max \left\{\left|\sum_{\substack{N_{\nu}<n \leqslant N_{\nu+1} \\
\lambda_{N_{\nu+1}}(n, m)<l(x)}} S_{n m}(x)\right|,\left|\sum_{\substack{N_{\nu}<n \leqslant N_{\nu+1} \\
\lambda_{N_{\nu+1}}(n, m) \geqslant l(x)}} S_{n m}(x)\right|\right\} \\
& \geqslant \frac{1}{2} \sum_{n=N_{\nu}+1}^{N_{\nu+1}} \frac{\mu(n)}{\rho\left(2^{2 n+1}\right)} \geqslant \frac{1}{2} \nu .
\end{aligned}
$$

Из (65) следует, что ряд $\sum_{k=1}^{\infty} a_{\sigma(k)} \psi_{\sigma(k)}(x)$ всюду неограниченно расходится. Теорема 4 доказана.

\section{§. Всюду расходящиеся ряды}

\section{по мультипликативным системам и смежные вопросы}

Пусть $p=\left(p_{1}, \ldots, p_{n}, \ldots\right)$ - последовательность целых чисел, $p_{n} \geqslant 2$, и пусть

$$
M(k)=\prod_{n=1}^{k} p_{n}, \quad M(0)=1 .
$$

Каждое $x \in[0,1)$ может быть представлено в виде

$$
x=\sum_{k=1}^{\infty} \frac{x_{k}}{M(k)}, \quad 0 \leqslant x_{k}<p_{k} .
$$

Если имеется два таких представления, то выбирается представление с конечным числом членов. Для $k=1,2, \ldots$ определим функции

$$
\varphi_{k-1}(x)=\exp \left(2 \pi i \frac{x_{k}}{p_{k}}\right) .
$$

Из (66)-(68) следует, что на $[0,1)$ сушествует $M(k)$ замкнутых слева интервалов постоянства функции $\varphi_{k-1}(x)$.

Каждое $n=0,1,2, \ldots$ имеет единственное представление

$$
n=\sum_{k=0}^{\infty} \alpha_{k} M(k), \quad \text { где } 0 \leqslant \alpha_{k}<p_{k+1} .
$$

Определим систему функций $\left\{\chi_{n}\right\}_{n=0}^{\infty}$ при $n \geqslant 1\left\{\chi_{0}=1\right\}$ следуюшим образом (см. [31], [32]):

$$
\chi_{n}(x)=\prod_{k=0}^{\infty}\left(\varphi_{k}(x)\right)^{\alpha_{k}} .
$$

$\left\{\chi_{n}\right\}$ - это полная система характеров счетного прямого произведения циклических групп различных порядков, отображенного на единичньй интервал посредством сохраняюшего меру преобразования. Система $\left\{\chi_{n}\right\}$ назьвается ограниченной, если

$$
\sup _{n} p_{n}=d<\infty .
$$


ТЕОРема 5. Пусть $\left\{\chi_{n}\right\}$ - ограниченная мультипликативная система, и пусть возрастающая последовательность положстельных чисел $w(n)$ удовлетворяет условию

$$
\sum_{n=1}^{\infty} \frac{1}{n w(n)}=\infty .
$$

Тогда существует последовательность комплексных чисел $\left\{c_{n}\right\}$, удовлетворяющая соотношению

$$
\sum_{n=1}^{\infty}\left|c_{n}\right|^{2} w(n)<\infty,
$$

и существует перестановка натурального ряда $\sigma(n)$ такая, что ряд

$$
\sum_{n=1}^{\infty} c_{\sigma(n)} \chi_{\sigma(n)}(x)
$$

всюду расходится.

ДоКАЗАТЕльСТво. Используя соотношения (71), (72), возьмем убывающую последовательность положительных чисел $\left\{\mu_{n}\right\}$ и возрастающую последовательность номеров $\left\{N_{\nu}\right\}$, а также выберем возрастаюшую последовательность натуральных чисел $l_{n} \in\left[0, N_{\nu+1}-N_{\nu}\right)(n=0,1, \ldots, 2 q(\nu)+1$ для некоторого $q(\nu))$ и целое число $p(\nu) \in[2, d]$, для которых вьполняются следуюшие соотношения (см. (66)):

$$
\begin{gathered}
\sum_{n=1}^{\infty} \frac{\mu^{2}(n)}{w(M(n))}<\infty, \\
p_{N_{\nu}+l_{n}}=p(\nu), \quad n=0,1, \ldots, 2 q(\nu)+1, \\
\sum_{n=0}^{q(\nu)} \frac{\mu\left(N_{\nu}+l_{2 n+1}\right)}{w\left(M\left(N_{\nu}+l_{2 n+1}\right)\right)} \geqslant C(d) \nu,
\end{gathered}
$$

где $C(d)$ - положительная постоянная.

Для каждого $\nu=1,2, \ldots$ определим подгруппы $G_{n m}^{(\nu)}$ функций $\left\{\chi_{k}\right\}$ и смежные классы по этим подгруппам $H_{n m}^{(\nu)}$. Положим

$$
H_{n m}^{(\nu)}=g_{n m}^{(\nu)} G_{n m}^{(\nu)}, \quad n=0,1, \ldots, q(\nu), \quad m=0,1, \ldots,(p(\nu))^{n}-1,
$$

где

$$
g_{n 0}^{(\nu)}=\varphi_{N_{\nu}+l_{2 n}}, \text { если } m=0,
$$

а при

$$
\begin{gathered}
m=\alpha_{1}(p(\nu))^{s_{1}}+\cdots+\alpha_{h(m)}(p(\nu))^{s_{h(m)}} \\
0 \leqslant s_{1}<s_{2}<\cdots<s_{h(m)}<n, \quad 1 \leqslant \alpha_{j}<p(\nu),
\end{gathered}
$$

положим

$$
g_{n m}^{(\nu)}=\varphi_{N_{\nu}+l_{2 n}} \varphi_{\beta(1)}^{\alpha_{1}} \varphi_{\beta(2)}^{\alpha_{2}} \cdots \varphi_{\beta(h(m))}^{\alpha_{h(m)}},
$$

где $\beta(j)=N_{\nu}+l_{2 s_{j}+1}$. 
Определим подгрупшы $G_{n m}^{(\nu)}$ при $n \geqslant 1\left(G_{00}^{(\nu)}=\chi_{0}\right)$ как прямое произведение циклических групп порядка $p(\nu)$ с образуюшими

$$
g_{n m k}^{(\nu)}=\varphi_{N_{\nu}+l_{2(n-k)}} \varphi_{\beta(1, k)}^{\alpha_{1}} \varphi_{\beta(2, k)}^{\alpha_{2}} \cdots \varphi_{\beta(h(m), k)}^{\alpha_{h(m)}},
$$

где при $k \leqslant s_{j}$

$$
\beta(j, k)=N_{\nu}+l_{2\left(s_{j}-k\right)+1},
$$

а если $k>s_{j}$, то полагаем по определению

$$
\varphi_{\beta(j, k)}=\chi_{0} .
$$

В силу соотношений (77)-(79) множества $H_{n m}^{(\nu)}$ попарно не пересекаются. Доказательство этого факта изложено в [33].

Для $n=0,1, \ldots, q(\nu), m=0,1, \ldots,(p(\nu))^{n}-1$ определим множества $E_{m}^{(n)}$. Положим $E_{0}^{(0)}=[0,1)$ и при $n \geqslant 1$ положим

$$
E_{p(\nu) m+r}^{(n+1)}=\left\{x \in E_{m}^{(n)} ; g_{n m}^{(\nu)}(x)=\exp \left(2 \pi i \frac{r}{p(\nu)}\right)\right\}
$$

где $r=0,1, \ldots, p(\nu)-1$.

Соотношения (77)-(80) означают, что подгрупшы $G_{n m}^{(\nu)}$ и множества $E_{m}^{(n)}$ обладают определенной наследственной структурой, а именно

$$
E_{m}^{(n)} \subset \cdots \subset E_{m_{k}}^{(n-k)} \subset \cdots \subset E_{0}^{(0)}
$$

где $m_{k}=\left[\frac{m}{(p(\nu))^{k}}\right]$, и, кроме того, выполняется равенство

$$
\operatorname{mes} E_{m}^{(n)}=(p(\nu))^{-n}
$$

Из определения классов $H_{n m}^{(\nu)}$ и множеств $E_{m}^{(n)}$ следует также, что каждая функция из подгрупшы $G_{n m}^{(\nu)}$ принимает постоянное значение на множестве $E_{m}^{(n)}$, а каждая $\chi_{k} \in$ $H_{n m}^{(\nu)}$ принимает постоянное значение на множестве $E_{p(\nu) m+r}^{(n+1)}, 0 \leqslant r<p(\nu)$.

В частности, если $x \in E_{p(\nu) m}^{(n+1)}$, то для некоторого $0 \leqslant \alpha(k)<p(\nu)$

$$
\chi_{k}(x)=\exp \left(2 \pi i \frac{\alpha(k)}{p(\nu)}\right)
$$

Представляя функцию $\chi_{k} \in H_{n m}^{(\nu)}$ в виде

$$
\chi_{k}=g_{n m}^{(\nu)} g_{n m 1}^{\gamma_{1}} g_{n m 2}^{\gamma_{2}} \cdots g_{n m n}^{\gamma_{n}},
$$

где $0 \leqslant \gamma_{j}<p(\nu)$, и учитьвая $(80)$ и $(83)$, заключаем, что при $x \in E_{p(\nu) m+r}^{(n+1)}$ справедливо равенство

$$
\exp \left(-2 \pi i \frac{\alpha(k)}{p(\nu)}\right) \chi_{k}(x)=\exp \left(2 \pi i \frac{r}{p(\nu)}\right) .
$$


Обозначим

$$
A_{n m}^{(\nu)}=\left\{k ; \chi_{k} \in H_{n m}^{(\nu)}\right\} .
$$

Определим теперь коэффициенты $\left\{c_{k}\right\}$. При $M\left(N_{\nu}\right) \leqslant k<M\left(N_{\nu+1}\right)$ положим

$$
c_{k}=\left\{\begin{array}{l}
\exp \left(-2 \pi i \frac{\alpha(k)}{p(\nu)}\right) \frac{\mu\left(N_{\nu}+l_{2 n+1}\right)}{(p(\nu))^{n} w\left(M\left(N_{\nu}+l_{2 n+1}\right)\right)} \\
0 \text { при остальных } k \in\left[M\left(N_{\nu}\right), M\left(N_{\nu+1}\right)\right) .
\end{array}\right.
$$

Из соотношений (74), (77)-(81) и (85), (86) следует, что

$$
\begin{aligned}
& \sum_{k=0}^{\infty}\left|c_{k}\right|^{2} w(k)=\sum_{\nu=1}^{N} \sum_{n=0}^{q(\nu)} \sum_{m=0}^{(p(\nu))^{n}-1} \sum_{\substack{(\nu) \\
k \in A_{n m}}}\left|c_{k}\right|^{2} w(k) \\
& \leqslant \sum_{\nu=1}^{\infty} \sum_{n=0}^{q(\nu)} w\left(M\left(N_{\nu}+l_{2 n+1}\right)\right) \sum_{m=0}^{(p(\nu))^{n}-1} \sum_{k \in A_{n m}^{(\nu)}}\left|c_{k}\right|^{2} \\
& \leqslant \sum_{\nu=1}^{\infty} \sum_{n=0}^{q(\nu)} \frac{\mu^{2}\left(N_{\nu}+l_{2 n+1}\right)}{w\left(M\left(N_{\nu}+l_{2 n+1}\right)\right)}<\infty .
\end{aligned}
$$

Следовательно, условие (73) выполнено.

Введем функции (см. (85))

$$
S_{n m}^{(\nu)}(x)=\sum_{k \in A_{n m}^{(\nu)}} c_{k} \chi_{k}(x)
$$

В силу равенства (84), имеем (см. (86), (87))

$$
S_{n m}^{(\nu)}(x)=\frac{\mu\left(N_{\nu}+l_{2 n+1}\right)}{w\left(M\left(N_{\nu}+l_{2 n+1}\right)\right)} \exp \left(2 \pi i \frac{r}{p(\nu)}\right),
$$

если $x \in E_{p(\nu) m+r}^{(n+1)}$.

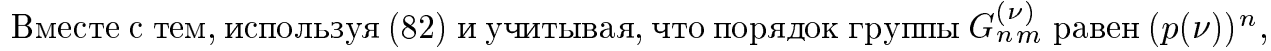
получаем

$$
\int_{E_{m}^{(n)}}\left|S_{n m}^{(\nu)}(x)\right|^{2} d x=\left\|S_{n m}^{(\nu)}\right\|_{2}^{2}
$$

и, значит (см. (68)-(70)),

$$
\operatorname{supp} S_{n m}^{(\nu)}=E_{m}^{(n)}
$$

Определим теперь требуемую перестановку $\sigma(k)$ ряда $\sum_{k=0}^{\infty} c_{k} \chi_{k}(x)$. Для каждого $\nu=1,2, \ldots$ определим взаимно однозначное отображение $\lambda_{\nu}(n, m)$ пар $(n, m)$, $n=0,1, \ldots, q(\nu), m=0,1, \ldots,(p(\nu))^{n}-1$, на отрезок натурального ряда

$$
\left[1, \frac{(p(\nu))^{q(\nu)+1}-1}{p(\nu)-1}\right]
$$


Положим

$$
\lambda_{\nu}(n, m)>\lambda_{\nu}\left(n, m_{1}\right), \text { если } m<m_{1} .
$$

Если $s<n$ и

$$
E_{m}^{(n)} \subseteq E_{q}^{(s+1)}
$$

где $q=p(\nu) l+k$, то положим

$$
\begin{aligned}
& \lambda(s, l-1)>\lambda(n, m)>\lambda(s, l) \text { при } k=0,1, \ldots,\left[\frac{p(\nu)}{2}\right]-1, \\
& \lambda(s, l)>\lambda(n, m)>\lambda(s, l+1) \text { при } k=\left[\frac{p(\nu)}{2}\right], \ldots, p(\nu)-1 .
\end{aligned}
$$

Для каждого фиксированного $\nu=1,2, \ldots$ расположим функции $S_{n m}^{(\nu)}(x)$ в порядке возрастания чисел $\lambda_{\nu}(n, m)$. Суммы

$$
\sum_{M\left(N_{\nu}\right) \leqslant k<M\left(N_{\nu+1}\right)} c_{k} \chi_{k}(x)
$$

как целое расположим в порядке возрастания номера $\nu$.

Установим, что справедливо следующее утверждение. Пусть

$$
\lambda_{\nu}\left(n_{1}, m_{1}\right)>\lambda_{\nu}(n, m)
$$

и при некотором $[p(\nu) / 2] \leqslant q<p(\nu)$ выполняется соотношение

$$
E_{p(\nu) m+q}^{(n+1)} \cap E_{m_{1}}^{\left(n_{1}\right)} \neq \varnothing .
$$

Тогда $n_{1}<n$ и

$$
E_{p(\nu) m+q}^{(n+1)} \subset E_{p(\nu) m_{1}+l}^{\left(n_{1}+1\right)}
$$

при некотором $[p(\nu) / 2] \leqslant l<p(\nu)$.

Докажем, что $n_{1}<n$. В самом деле, если $n_{1}=n$, то из (80), (81), (93) следует $m_{1}=m$, а это противоречит (92). Пусть теперь $n_{1}>n$. Тогда из (93) следует, что

$$
E_{m_{1}}^{\left(n_{1}\right)} \subseteq E_{p(\nu) m+q}^{(n+1)}
$$

и в силу соотношения (91) имеем

$$
\lambda_{\nu}(n, m)>\lambda_{\nu}\left(n_{1}, m_{1}\right)
$$

что противоречит (92).

Итак, $n_{1}<n$ и, следовательно (см. (80), (81), (93)),

$$
E_{m}^{(n)} \subseteq E_{p(\nu) m_{1}+l}^{\left(n_{1}+1\right)}
$$

при некотором $l=0,1, \ldots, p(\nu)-1$.

Применяя соотношения (90), (91) и учитьвая (92), находим (см. (95)), что $[p(\nu) / 2] \leqslant$ $l<p(\nu)$. Тем самьм утверждение доказано (см. (80), (94), (95)). 
Для каждого $x \in[0,1)$ определим номер $L_{\nu}(x)$ следующим образом:

$$
L_{\nu}(x)=\min \lambda_{\nu}(n, m),
$$

где минимум берется по таким парам $(n, m)$, для которых $x \in E_{p(\nu) m+q}^{(n+1)},[p(\nu) / 2] \leqslant$ $q<p(\nu)$.

Используя определение перестановки $\sigma(k)$, получаем (см. $(96))$

$$
\begin{aligned}
& \sum_{M\left(N_{\nu}\right) \leqslant k<M\left(N_{\nu+1}\right)} c_{\sigma(k)} \chi_{\sigma(k)}(x) \\
& =\sum_{\lambda_{\nu}(n, m)<L_{\nu}(x)} S_{n m}^{(\nu)}(x)+\sum_{\lambda_{\nu}(n, m) \geqslant L_{\nu}(x)} S_{n m}^{(\nu)}(x)=\sum^{(1)}+\sum^{(2)} .
\end{aligned}
$$

Применим доказанное утверждение (см. (92)-(94)) и используем определение (96). Имеем (см. (89)):

$$
\sum^{(1)}=\sum_{\lambda_{\nu}(n, m)<L_{\nu}(x)} S_{n m}^{(\nu)}(x), \quad x \in E_{p(\nu) m+q}^{(n+1)},
$$

где $0 \leqslant q<[p(\nu) / 2]$

$$
\sum^{(2)}=\sum_{\lambda_{\nu}(n, m) \geqslant L_{\nu}(x)} S_{n m}^{(\nu)}(x), \quad x \in E_{p(\nu) m+q}^{(n+1)},
$$

где $[p(\nu) / 2] \leqslant q<p(\nu)$.

Далее, для любой последовательности положительных чисел $\left\{a_{k}\right\}$ и любых натуральных $N$ и $p$ справедливо неравенство

$$
\left|\sum_{k=1}^{N} a_{k} \exp \left(2 \pi i \frac{q(k)}{p}\right)\right| \geqslant \cos \frac{\pi}{2}\left(1-\frac{1}{p}\right) \cdot \sum_{k=1}^{N} a_{k},
$$

если натуральные числа $q(k)$ удовлетворяют соотношению

$$
0 \leqslant q(k)<\left[\frac{p}{2}\right]
$$

или если $q(k)$ удовлетворяют неравенству

$$
\left[\frac{p}{2}\right] \leqslant q(k)<p .
$$

Объединяя соотношения $(71),(76),(88)$ и (98)-(100), находим, что

$$
\begin{aligned}
\left|\sum^{(1)}\right|+\left|\sum^{(2)}\right| & \geqslant \cos \frac{\pi}{2}\left(1-\frac{1}{d}\right) \cdot \sum_{n=0}^{q(\nu)} \frac{\mu\left(N_{\nu}+l_{2 n+1}\right)}{w\left(M\left(N_{\nu}+l_{2 n+1}\right)\right)} \\
& \geqslant C(d) \cos \frac{\pi}{2}\left(1-\frac{1}{d}\right) \cdot \nu .
\end{aligned}
$$

Отсюда следует (см. (97), (100)), что ряд $\sum_{k=0}^{\infty} c_{\sigma(k)} \chi_{\sigma(k)}(x)$ неограниченно расходится при любом $x \in[0,1)$. Теорема 5 доказана.

Приведем теперь оценки снизу мажоранты частных сумм для полиномов по переставленньм мультипликативным системам (см. [33]). 
Теорема 6. Пусть $\left\{\chi_{n}\right\}$ - ограниченная мультипликативная система. Тогда для любого $N=2,3, \ldots$ существует перестановка $\sigma(n)$ чисел $n=0,1, \ldots, N$ такая, что

$$
\int_{0}^{1} \max _{m \leqslant N}\left|\sum_{n=0}^{m} \chi_{\sigma(n)}(x)\right|^{2} d x \geqslant C(d) N \log N
$$

ТЕОрема 7. Пусть $\left\{\chi_{n}\right\}$ - ограниченная мультипликативная система. Для любого $N=2,3, \ldots$ существует полином $P_{N}(x)=\sum_{n=0}^{N} a_{n} \chi_{n}(x)$ такой, что

$$
\left|P_{N}(x)\right|=1 \quad \text { nри всеx } \quad x \in[0,1),
$$

и существует перестановка $\sigma(n)$ чисел $n=0,1, \ldots, N$, для которой

$$
\operatorname{mes}\left\{\max _{m \leqslant N}\left|\sum_{n=0}^{m} a_{\sigma(n)} \chi_{\sigma(n)}(x)\right|>\alpha \sqrt{\log N}\right\} \geqslant \gamma
$$

где $\alpha, \gamma$ - положительные постояннье, зависящие только от $d$.

Автор предполагает, что теоремы 5-7 являются точными для обших мультипликативных систем.

Конструкшия, использованная для доказательства теоремы 7 , была применена для доказательства следующего утверждения (см. [34], [35]).

ТЕОРЕМа 8. Пусть модуль непрерывности удовлетворяет условию

$$
\sum_{n=2}^{\infty} \frac{\omega\left(\frac{1}{n}\right)}{n \sqrt{\log n}}=\infty
$$

Тогда для любой ограниченной мультипликативной системы $\left\{\chi_{k}\right\}$ существует непрерывная функция $f \in H^{\omega}$ такая, что

$$
f(x) \sim \sum_{k=0}^{\infty} c_{k} \chi_{k}(x), \quad c_{k}=\int_{0}^{1} f(x) \bar{\chi}_{k}(x) d x,
$$

и существует перестановка натурального ряда $\sigma(k)$, для которой ряд

$$
\sum_{k=0}^{\infty} c_{\sigma(k)} \chi_{\sigma(k)}(x)
$$

расходится почти всюду.

Теоремы 5 и 8 являются в настоящее время наиболее сильными результатами о безусловной сходимости почти всюду рядов Фурье по мультипликативным системам и, в частности, по системе Уолша. Отметим, что для тригонометрической системы сейчас нет результатов о сушествовании функций из классов $H^{\omega}$ с рядами, расходящимися на множестве полной меры после перестановки.

Автором также установлены два утверждения, относяшиеся к оценке сверху мажоранты частных сумм рядов по переставленным системам характеров (см. [34], [36]). 
ТЕОРема 9. Пусть $\left\{\chi_{n}\right\}$ - ограниченная мультипликативная система, и пусть функция $n(x)$ принимает целье положстельные значения, $\|n(x)\|_{\infty}<M(q)$ при некотором $q=1,2, \ldots u$

$$
n(x)=\sum_{j=1}^{N} m_{j}(x),
$$

где функции $m_{j}(x)$ имеют непересекаюшиеся носители и каждая их них монотонна на своем носителе. Тогда неравенство

$$
\left\|\sum_{k=0}^{n(x)} c_{k} \chi_{\sigma(k)}(x)\right\|_{1} \leqslant B(d) \sqrt{N}\|c\|_{2}
$$

выполняется для всех перестановок $\sigma(k)$ чисел $k=0,1, \ldots, M(q)-1$ и для любой последовательности комплексных чисел $c=\left\{c_{k}\right\}$.

Из теоремы 9 следует, что оценка типа результата Л. Карлесона имеет место для любой перестановки ограниченной мультипликативной системы, если мажоранта монотонна. При доказательстве теоремы 9 использовались результаты Р. Ханта и М. Тейблесона [9] и Дж. Госселина и У. Янга [37].

Следуюшая теорема показывает, что для оценки мажоранты частных сумм рядов по переставленным мультипликативным системам достаточно иметь оценку мажоранты переставленного ядра Дирихле.

ТеОРема 10. Пусть $\left\{\chi_{n}\right\}$ - мультипликативная система, $q$ - натуральное число и $\sigma(n)$ - перестановка чисел $n=0,1, \ldots, M(q)-1$. Тогда для любой последовательности комплексных чисел $c=\left\{c_{n}\right\}$ выполняется неравенство

$$
\begin{aligned}
\max _{\sigma} \| & \max _{m<M(q)}\left|\sum_{n=0}^{m} c_{n} \chi_{\sigma(n)}(x)\right| \|_{1} \\
& \ll(M(q))^{-\frac{1}{2}}\|c\|_{2} \max _{\sigma}\left\|\max _{m<M(q)}\left|\sum_{n=0}^{m} \chi_{\sigma(n)}^{(1)}(x)\right|\right\|_{2},
\end{aligned}
$$

где $\left\{\chi_{n}^{(1)}\right\}-$ мультипликативная система, образованная последовательностью $\left\{p_{q-j+1}\right\}, j=1, \ldots, q$.

Отметим, что в теореме 10 мультипликативная система $\left\{\chi_{n}\right\}$ не предполагается ограниченной.

\section{СПИСОК ЛИТЕРАТУРЫ}

[1] A. N. Kolmogoroff. Une série de Fourier-Lebesgue divergente presque partout // Fund. Math. 1923. V. 4. Р. 324-328. (Рус. пер.: Ряд Фурье-Лебега, расходящийся почти всюду // А.Н. Колмогоров. Избранные труды. Математика и механика. М.: Наука, 1985. С. 8-11.)

[2] A. N. Kolmogoroff. Une série de Fourier-Lebesgue divergente partout // C. R. Acad. Sci. Paris. 1926. V. 183. Р. 1327-1329. (Рус. пер.: Ряд Фурье-Лебега, расходящийся всюду // А.Н. Колмогоров. Избранные труды. Математика и механика. М.: Наука, 1985. С. 73-75.)

[3] A. N. Kolmogoroff, G. A. Seliverstoff. Sur la convergence des séries de Fourier // Atti Accad. Naz. Lincei Rend. 1926. V. 3. P. 307-310. (Рус. пер.: О сходимости рядов Фурье // А. Н. Колмогоров. Избранные труды. Математика и механика. М.: Наука, 1985. С. 69-73.) 
[4] A. N. Kolmogoroff, D. E. Menchoff. Sur la convergence des séries de fonctions orthogonales // Math. Z. 1927. V. 26. № 2/3. P. 432-441. (Рус. пер.: О сходимости ортогональных рядов // А.Н. Колмогоров. Избранные труды. Математика и механика. М.: Наука, 1985. C. $75-85$.)

[5] D. E. Menchoff. Sur la convergence des séries de fonctions orthogonales. I // Fund. Math. 1923. V. 4. P. $82-105$.

[6] D.E. Menchoff. Sur la sommation des séries de fonctions orthogonales par des méthodes de Cesaro // Матем. сб. 1940. T. 8 (50). № 1. C. 121-136.

[7] L. Carleson. On convergence and growth of partial sums of Fourier series // Acta Math. 1966. V. 116. P. 135-157.

[8] R. Hunt. Almost everywhere convergence of Walsh-Fourier series of $L^{2}$ functions // Actes du Congrés International des Mathématiciens (Nice, 1970). V. 2. Paris: Gauthier-Villars, 1971. P. 655-661.

[9] R. Hunt, M. Taibleson. Almost everywhere convergence of Fourier series on the ring of integers of a local field // SIAM J. Math. Anal. 1971. V. 2. № 4. P. 607-625.

[10] P. Sjölin. An inequality of Paley and convergence a.e. of Walsh-Fourier series // Ark. Mat. 1969. V. 7. P. 551-570.

[11] J. Gosselin. Almost everywhere convergence of Vilenkin-Fourier series // Trans. Amer. Math. Soc. 1973. V. 185. P. 345-370.

[12] P. Sjölin, F. Soria. Remarks on a theorem by N. Y. Antonov // Studia Math. (to appear).

[13] E. Stein. On limit of sequences of operators // Ann. of Math. (2). 1961. V. 74. P. 140-170.

[14] F. Schipp. Über die Divergenz der Walsh-Fourierreihen // Ann. Univ. Sci. Budapest. Eötvös Sec. Math. 1969. V. 12. P. 49-62.

[15] F. Schipp. On boundedly divergent Walsh-Fourier series // Acta Math. Hungar. 1990. V. 56. №3-4. P. 361-367.

[16] K. Moon. An everywhere divergent Fourier-Walsh series of the class $L\left(\log ^{+} \log ^{+} L\right)^{1-\varepsilon} / /$ Proc. Amer. Math. Soc. 1975. V. 50. P. 309-314.

[17] P. Simon. On the divergence of Vilenkin-Fourier series // Acta Math. Hungar. 1983. V. 41. № 3-4. P. 359-370.

[18] С. В. Бочкарев. Расходящийся на множестве положительной меры ряд Фурье для произвольной ограниченной ортонормированной системы // Матем. сб. 1975. Т. 98. № 3. C. $436-449$

[19] К. С. Казарян. О некоторых вопросах теории ортогональных рядов // Матем. сб. 1982. T. 119. № 2. C. 278-294.

[20] С. В. Бочкарев. Всюду расходящиеся ряды Фурье-Уолша // Докл. РАН. 2003. Т. 390. № 1. C. 11-14.

[21] П. Л. Ульянов. Расходящиеся ряды по системе Хаара и по базисам // Докл. АН СССР. 1961. Т. 138. № 3. С. 556-559.

[22] А. М. Олевский. Расходящиеся ряды из $L_{2}$ по полным системам // Докл. АН СССР. 1961. Т. 138. № 3. С. 545-548.

[23] С. В. Бочкарев. О мажоранте частных сумм для переставленной системы Уолша // Докл. АН СССР. 1978. Т. 239. № 3. С. 509-510.

[24] С. В. Бочкарев. Метод усреднений в теории ортогональных рядов // Proceedings of the International Congress of Mathematicians (Helsinki, 1978) / ed. O. Lehto. V. 2. Helsinki: Univ. of Helsinki, 1979. P. 598-604.

[25] С. В. Бочкарев. Перестановки рядов Фурье-Уолша // Изв. АН. Сер. матем. 1979. Т. 43. № 5. C. $1025-1041$.

[26] S. Nakata. On the unconditional convergence of Walsh series // Anal. Math. 1979. V. 5. № 3. P. 201-205.

[27] F. Schipp, W. Wade, P. Simon. Walsh Series. An Introduction to Dyadic Harmonic Analysis. Bristol: Hilger, 1990.

[28] В. В. Петров. Предельные теоремы для сумм независимых случайных величин. М.: Наука, 1987.

[29] S. V. Konyagin. On divergence of trigonometric Fourier series everywhere // C. R. Acad. Sci. Paris Sér. I Math. 1999. V. 329. № 8. P. 693-697. 
[30] С. В. Бочкарев. О проблеме гладкости функций, ряды Фурье-Уолша которых расходятся почти всюду // Докл. РАН. 2000. Т. 371. №6. С. 730-733.

[31] J. Price. Certain groups of orthonormal step functions // Canad. J. Math. 1957. V. 9. № 3. P. 413-425.

[32] Г.Н. Агаев, Н.Я. Виленкин, Г. М. Джафарли, А.И. Рубинштейн. Мултипликативные системы функций и гармонический анализ на нуль-мерных группах. Баку: Элм, 1981.

[33] С. В. Бочкарев. Оценки некоторых сумм характеров // Труды МИАН. 1994. Т. 207. C. $42-53$.

[34] С. В. Бочкарев. Перестановки мультипликативных систем // Докл. АН СССР. 1991. T. 318. C. $521-524$.

[35] С. В. Бочкарев. Почти всюду расходящиеся перестановки рядов Фурье по мулштипликативным системам функций классов $H^{\omega} / /$ Труды МИАН. 1992. Т. 201. С. 115-130.

[36] S. V. Bochkarev. Rearrangements of periodic multiplicative orthogonal series // Colloq. Math. 1990. V. 60/61. № 1. P. 291-299.

[37] J. Gosselin, W. Young. On rearrangements of Vilenkin-Fourier series which preserve almost everywhere convergence // Trans. Amer. Math. Soc. 1975. V. 209. P. 157-174.

Математический институт им. В. А. Стеклова РАН

Поступила в редакцию

E-mail: bochkarv@mi.ras.ru

20.11 .2003 